\title{
Need of Removing Delivered Message Replica from Delay Tolerant Network - A Problem Definition
}

\author{
Harminder Singh Bindra ${ }^{1}$, A L Sangal ${ }^{2}$ \\ ${ }^{1}$ Research Scholar, Department of Computer Science \& Engineering, DR B R Ambedkar National Institute of \\ Technology, Jalandhar \\ ${ }^{2}$ Director, DAV Institute of Technology, Jalandhar
}

\begin{abstract}
Recent wireless networks observe number of deployments in various conditions where they come across different intensities of link disconnection. On the basis of extent of the operating circumstances these networks are termed as Intermittently Connected Networks (ICNs). The prevailing TCP/IP protocol cannot be operational in ICNs thus providing number of new stimulating problems that are appealing the focus of the researchers. The multi-copy routing schemes achieve higher delivery probability as compared to the single copy routing scheme. This improvement is achieved at the cost of higher resource utilization i.e. multi-copy routing protocols requires more buffer space, more bandwidth, incur more overheads and consume other vital network resources. Contribution of this work is the deletion of useless replicas of the messages which are already delivered to the intended destination. We evaluate our proposed method by simulation, on four major DTNs routing algorithms: Epidemic, Spray and Wait, ProPHET and MaxProp.
\end{abstract}

Index Terms - Delay Tolerant Network, MaxProp, Epidemic, Spray and Wait, Prophet, Delay

\section{INTRODUCTION}

As technology advances at ostensibly inexorable stride, the world we sentient in is changing. In today's the world, we are regulars of not only goods and services, but information. The potentials obsessed by technology and the Internet have led to an aptitude to yield and munch an unparalleled volume of information through a varied variety of channels - computing anywhere, perhaps everywhere, and at any time. With the satiety and growing sophistication of personal mobile devices, and short-range wireless communications included on phones, MP3 players, games consoles, and other sensors, there are countless prospects to create, publish, and ingest information. Taking benefit of the direct contacts which occur when these devices are in close vicinity allows us to share and dispense information in new ways, exploiting the capacity existing at the boundaries of the network. There are also states where immobile infrastructure is entirely or partly absent and communication can only be sustained with an ad hoc paradigm. In pushing communication into increasingly incoherent (disconnected) regions and challenging locations such as inaccessible villages or into the realm of space, we face new demands in the establishment of endto-end communication: information must be tolerant of possibly extended delays and networks may be recurrently disconnected[1]. Messages must be buffered at nodes while we await upcoming contacts or mobile nodes cross disconnections, carrying messages amongst isolated clusters.

Under challenged networks, there is raised level of inherent uncertainty and any arbitrary node totally lacks network state information (i.e. information about other nodes in the network, network topology, etc.) and thus has to formulate its own operating decisions. A network of this type is clearly not appropriate to the provision of real-time information such as voice or video, rather it is well-matched to provision of information which is tolerant of delay to a certain degree.

Delay-/Disruption-Tolerant Networking is an overlay architecture intended to operate above the protocol stacks of the distinct ICNs and enable gateway functionality between them through the use of storage capacity, a variety of protocol techniques, replication and parallel forwarding, forward error correction and many other techniques for overcoming communication impairments[1].

Among the two categories of DTN routing schemes, the multi-copy routing schemes achieve higher delivery probability as compared to the single copy routing scheme. This improvement is achieved at the cost of higher resource utilization i.e. multi-copy routing protocols requires more buffer space, more bandwidth, incur more overheads and consume other vital network resources[2].

It is evident from the literature that most of the work in the field of routing in delay tolerant network is related to designing and formulating the routing strategy which should use minimal amount of network information and should forward the message in efficient manner. Not much of the literature is found which deals with the messages copies which are left over in the network at the 
time of message delivery. These message copies still utilize the network resources and the nodes still continues to assume these as undelivered messages. So nodes continues to work upon these messages in the normal routing process which in turn replicate these messages copies in the network leading to occupancy of more network resources by these delivered message copies. These resources which are being held by the copies of delivered messages are of no use for the performance of the network rather they degrade the performance of the network. So the major gap being identified from the review of the literature related to the routing in DTN is existence of useless replicas (of delivered messages) in the network and no mechanism is there to deal with these replicas except the TTL expiry.

Mostly the routing protocols are self-integrated with the storage management techniques. For example, Hop based TTL is added to Spray and Wait[3], passive cure to PEAR [4]. Also there is vast amount of work already carried out by many researchers about the buffer management policies.

Our method basically relaxes resources to prevent exhaustion and take care of storage overflow problem in contrast to counter measures in work by Krifa [5]

The contribution of this work is the deletion of useless replicas of the messages which are already delivered to the intended destination. As these replicas continues to use the resources and contribute in further replication at the next contact opportunity. So these replicas consumes the valuable resources of the network like storage, bandwidth, energy of nodes etc. deleting these replicas relaxes these resources and thus this freed up resources can be utilized for the replication/communication of the undelivered messages in more efficient manner.

We evaluate our proposed method by simulation, on four major DTNs routing algorithms: Epidemic[6], Spray and Wait[3], ProPHET[7] and MaxProp[8] .

Section-II provides the background about message replication in the delay tolerant networks. In this we have explained the replication process with the help of example and shown how the replicas exists in the network even after the delivery of the message to the intended destination. In section-III we have carried out the hypothetical simulation in which we have deleted the all the replicas of the message as and when it is delivered to the intended destination. At present we are not concerned with the procedure of how to identify the replica and how to delete it, we have just dropped the message copies when any of the copy reached the destination. Section-IV concludes the paper.

\section{Message Replication In Delay Tolerant NETWORK}

In the Delay Tolerant Networks communication opportunities are intermittent. Network is partitioned and no continuous end-to-end path exists from source to destination. Over the course of time, link comes up and down. Due to occasional partial connectivity or node mobility, the sequence of connectivity graphs over a time interval are overlapped to get an end-to-end path from source to destination. It implies that a message could be sent over an existing link, get buffered at the next hop until the next link in the path comes up, and so on and so forth, until it reaches the final destination. This routing is often referred to as Store-Carry-Forward Routing[9].

The example below explains the above mentioned replication process and show the existence of replica after the delivery of bundle/message to the destination. Further we executed hypothetical simulation in which it has been deduced that there is scope of improvement in the performance of multi copy routing scheme if these replicas are removed from the network after the bundle has been delivered to the destination.

In the fig. 1 , at time $t 1$ source ' $S$ ' generates a message for the Destination ' $D$ '. At this point of time there exists different disjoint regions of networks and $\mathrm{S}$ is connected to only 1,2 and 3 nodes. At time t2, the source $\mathrm{S}$ forwards the message to the node 3 .

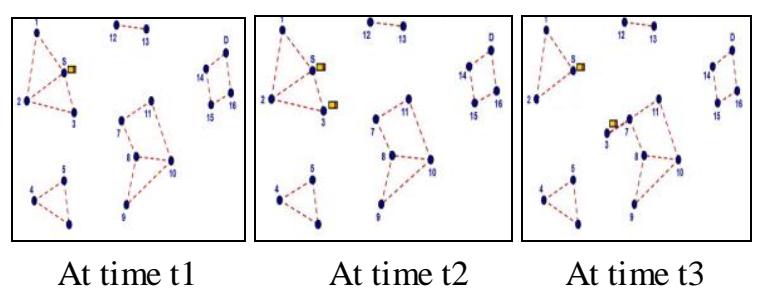

Fig. 1: Network and data bundle status at time instances $\mathrm{t} 1, \mathrm{t} 2$ and $\mathrm{t} 3$

At time instance $\mathrm{t} 3$, the node 3 moves to the new location and thus the node 3 come in contact of region with nodes $7,8,9,10$ and 11 . At time 4 , node 3 forwards the message to node 7 which in turn is forwarded to node 11(time instances t5). In this process the node $S, 3$ and 7 retains a copy of the forwarded bundle fig. 2 .

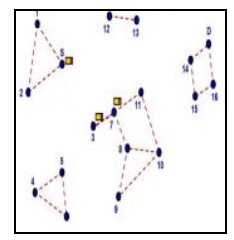

At time $\mathrm{t} 4$

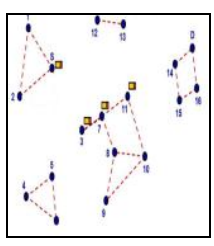

At time $\mathrm{t} 5$

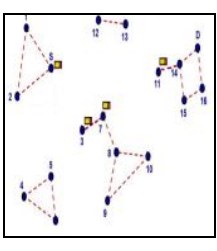

At time $t 6$
Fig. 2: Network and data bundle status at time instances $t 4, t 5$ and t6

Then node 11 at time t6 moves to new point in the network and thus comes in the communication range of node 14 (fig. 2). A link is established between 11 and 14 and thus node becomes the member of region with nodes $14,15,16$ and D.

The message is forwarded to node 14 by node 11 retaining the copy of the original message. This message is forwarded to node D (Destination) by node 14 again retaining the copy of it (time instance 7 fig. 3). At time t8 the message is delivered to the intended destination. But if we see the fig. 3 and the state of the network we can identify that in this process there exists 5 replicas of 
this delivered message which are now useless and are utilizing the resources. These messages will keep on utilizing the resources and will participate in further replications as these nodes are not aware of the delivery of the message to the final destination. So there is great need of removing these replicas from the network so as to free up the held resources. And this can be achieved if the delivery information is communicated back to these nodes.

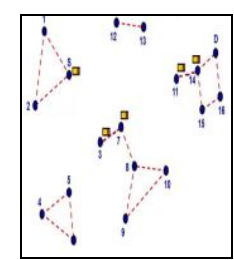

At time $\mathrm{t} 7$

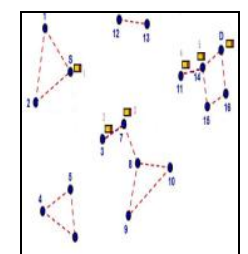

At time $\mathrm{t} 8$
Fig. 3: Network and data bundle status at time instances $t 7$ and t8

\section{PROBLEM Formulation}

In order to analyze the percentage scope of improvement in the performance of DTN routing protocols by deleting the replicas after delivering the bundle to destination, we have carried out a hypothetical simulation. In this simulation, at the time of delivery of message to the destination we have deleted all the replicas from the network and evaluated the performance in this scenario (at this point of time we are not concerned how to delete the replica so we have not focusing on procedure of deletion, we have just dropped the replicas from network). Then we compared this performance values to the base routing protocols performance values and evaluated the percentage scope of improvement in the performance.

\section{a) Simulation Setup}

The above mentioned simulation is carried out using Opportunistic Network Environment (ONE) simulator [10]. ONE is an agent-based discrete event simulation engine. At each simulation step the engine updates a number of modules that implement the main simulation functions. The main functions of the ONE simulator are the modeling of node movement, inter-node contacts, routing and message handling. Result collection and analysis are done through visualization, reports and postprocessing tools. The elements and their interactions are shown in figure 4.

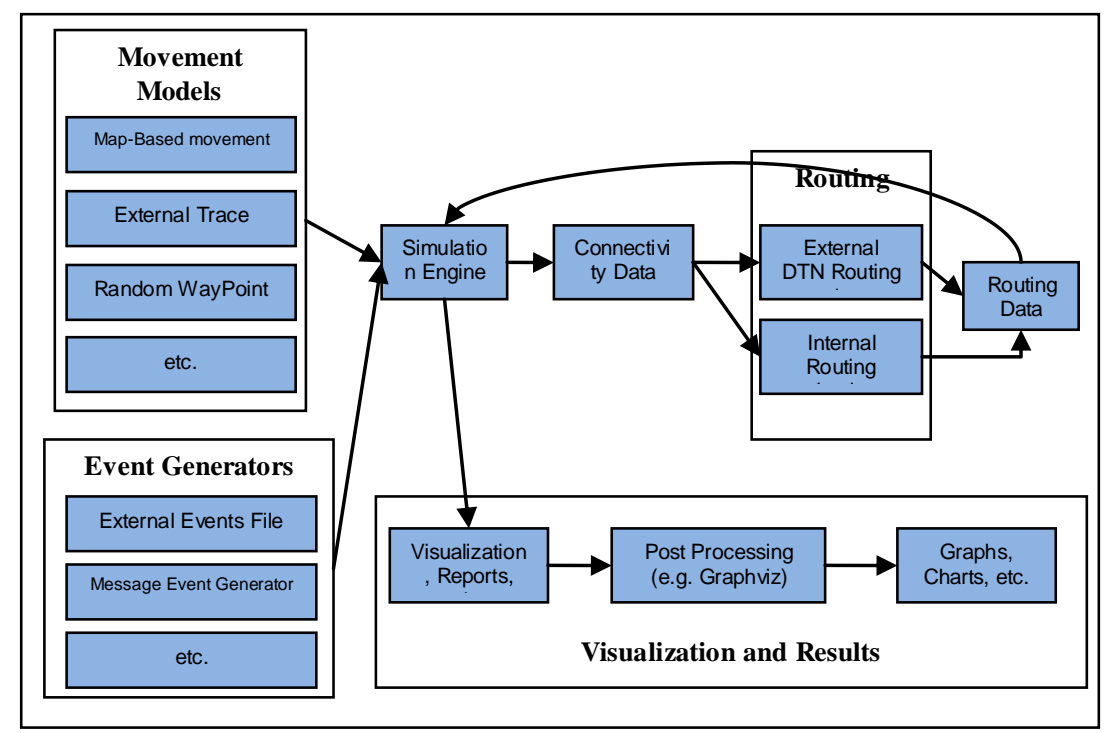

Fig. 4: Overview of ONE simulation environment.

The detailed simulations parameters are summarized in table .

Table 1: Simulation Parameter for the hypothetical simulation in which all replicas are deleted from the network when the mes sage is delivered to the destination.

\begin{tabular}{|c|c|c|c|}
\hline \multicolumn{4}{|c|}{ Scenario Setting } \\
\hline Name & simulateConnection & updateInterval & endTime \\
\hline Default_scenario & True & $0.1 \mathrm{~s}$ & $43200 \mathrm{~s}$ \\
\hline \multicolumn{2}{|c|}{ Interface Specific Setting } \\
\hline Name & Type & Transmit Speed & Transmit Range \\
\hline
\end{tabular}




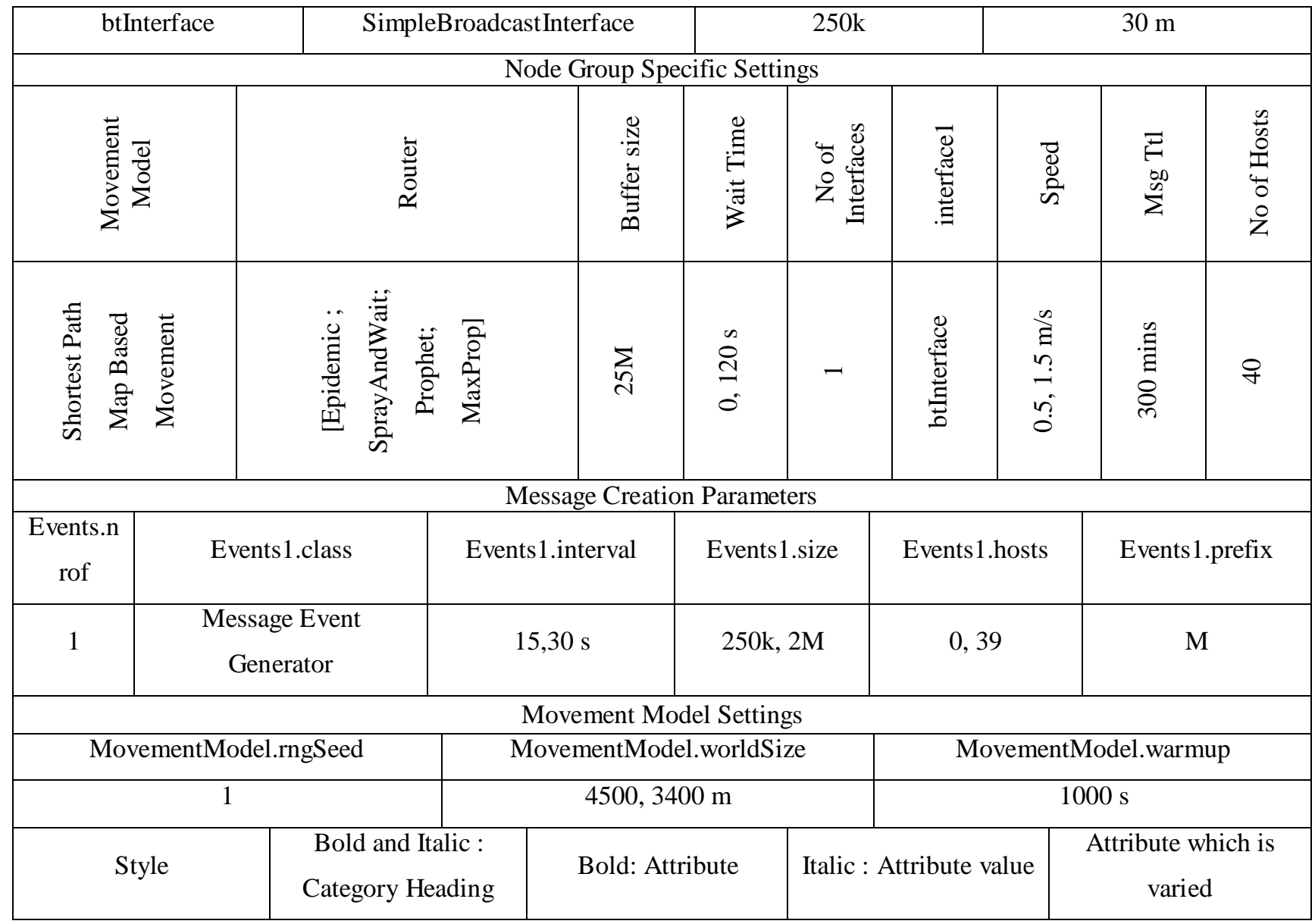

b) Results

The results obtained are summarized in the table 2 . We have analyzed that maximum scope of improvement is there in Prophet routing protocol. There we have seen $36 \%, 71 \%$, and $31 \%$ improvement in delivery probability, overhead ratio and average latency respectively. Further in Epidemic routing protocol there is $31 \%, 14 \%$ and $26 \%$ scope of improvement in delivery probability, overhead ratio and average latency respectively. The overall comparison is also depicted in graph Fig. 5.

Table 2: \% improvement in the performance of considered routing protocols when all replicas are deleted

\begin{tabular}{|c|c|c|c|c|c|c|c|c|c|}
\hline \multirow[b]{2}{*}{ Protocol } & \multicolumn{3}{|c|}{ Delivery Probability } & \multicolumn{3}{|c|}{ Overhead Ratio } & \multicolumn{3}{|c|}{ Average Latency } \\
\hline & 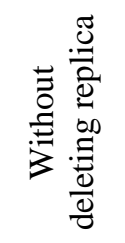 & 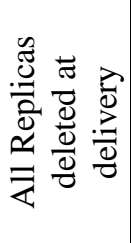 & 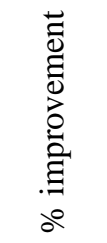 & 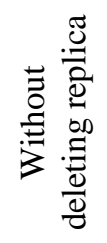 & 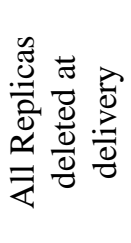 & 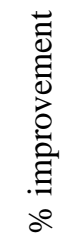 & 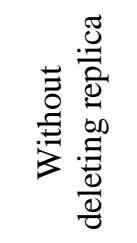 & 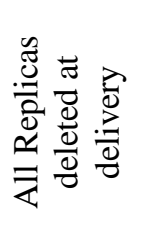 & 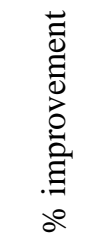 \\
\hline Epidemic & 0.71 & 0.93 & 31.76 & 26.56 & 13.80 & 48 & 2734.06 & 2014.76 & 26.30 \\
\hline $\begin{array}{c}\text { Spray And } \\
\text { Wait }\end{array}$ & 0.8105 & 0.93 & 15.91 & 15.94 & 5.99 & 62 & 2258.82 & 1570.71 & 30.46 \\
\hline MaxProp & 0.9657 & 0.96 & 0.25 & 9.69 & 8.84 & 8.77 & 1468.96 & 1359.92 & 7.42 \\
\hline Prophet & 0.6988 & 0.95 & 36.13 & 23.54 & 6.86 & 70.8 & 2585.92 & 1778.99 & 31.20 \\
\hline
\end{tabular}


From the results it is evident that when the replicas of the delivered messages are deleted from the network the delivery probability, overhead ratio and average latency of the all the considered routing protocols improves by a great extent. These results motivate us to design a mechanism to delete these replicas from the network as soon as the data message is delivered to the intended destination.

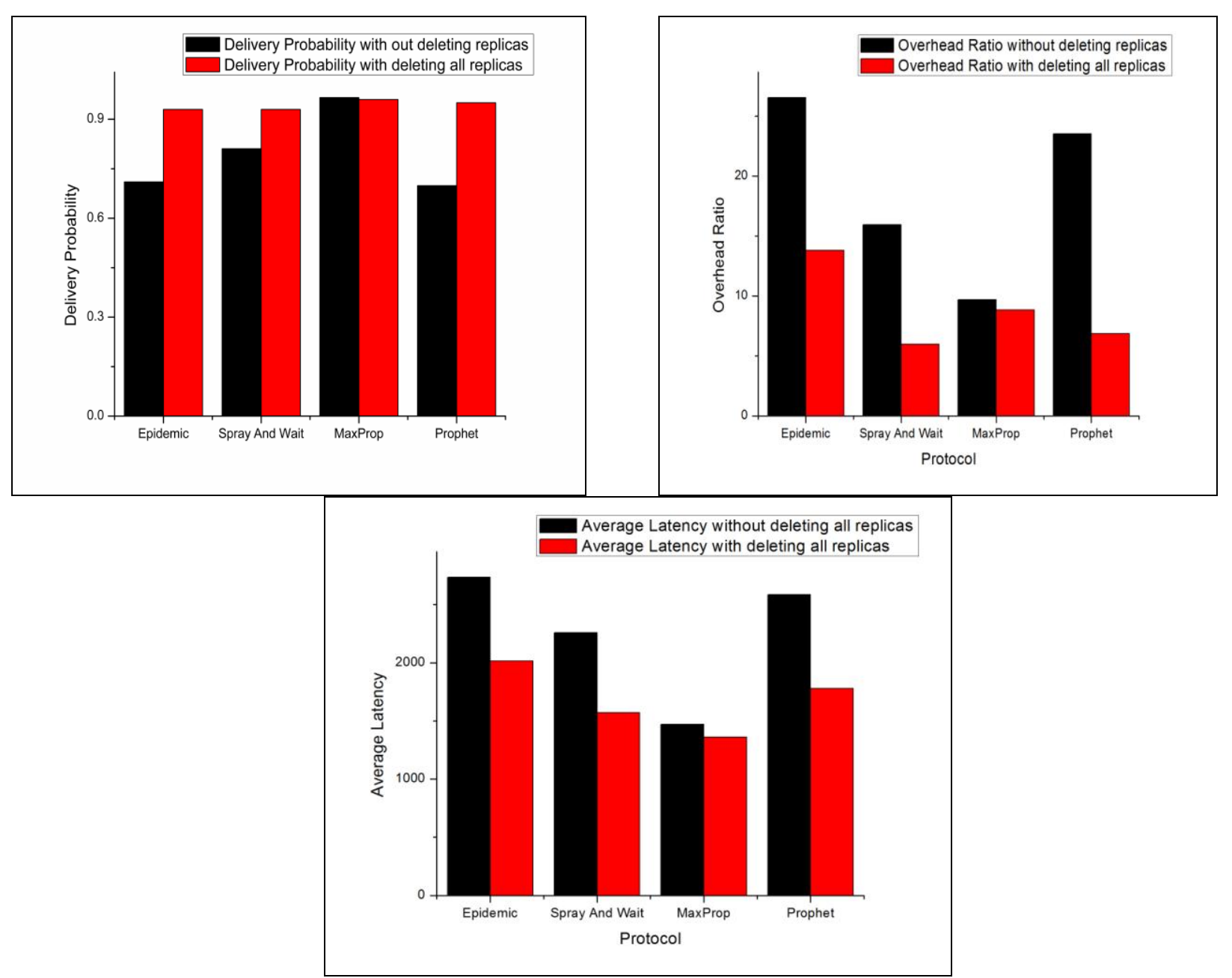

Fig. 5: Comparison of scope of improvement in routing protocols performance when all the replicas are deleted after delivery of message to the destination.

\section{CONCLUSIONS \& RECOMMENDATIONS}

In this paper we have tried to identify problems associated with the existence of the replicas of the already delivered messages. Also we have estimated percentage scope of improvement in performance of Delay Tolerant Routing Protocols by deleting the replicas of the delivered messages from the network. It is found that if the replicas are deleted at the same time when the data bundle is delivered then there is maximum performance improvement in PROPHET routing protocols. The Epidemic, Spray and Wait routing protocols also showed the performance improvement. There was marginal improvement in the MaxProp routing protocol. In future work we are working to define mechanism for the deletion of these replicas from the network.

\section{REFERENCES}

[1] M. Khabbaz, C. Assi, and W. Fawaz, "DisruptionTolerant Networking: A Comprehensive Survey on Recent Developments and Persisting Challenges," Communications Surveys \& Tutorials, IEEE, vol. PP, no. 99, pp. 1-34, 2011.

[2] E. Bulut, "OPPORTUNISTIC ROUTING ALGORITHMS IN DELAY TOLERANT NETWORKS," COMPUTER SCIENCE, Rensselaer Polytechnic Institute, New York, 2011.

[3] T. Spyropoulos, K. Psounis, and C. S. Raghavendra, "Spray and wait: an efficient routing scheme for intermittently connected mobile networks," in Proceedings of the 2005 ACM SIGCOMM workshop on Delay-tolerant networking, Philadelphia, Pennsylvania, USA, 2005, pp. 252-259. 
[4] H. Ochiai, and H. Esaki, "Mobility entropy and message routing in community-structured delay tolerant networks," in Proceedings of the 4th Asian Conference on Internet Engineering, Pratunam, Bangkok, Thailand, 2008.

[5] A. Krifa, C. Baraka, and T. Spyropoulos, "Optimal Buffer Management Policies for Delay Tolerant Networks." pp. 260-268.

[6] A. Vahdat, and D. Becker, "Epidemic Routing for Partially Connected Ad Hoc Networks," 2000.

[7] A. Lindgren, A. Doria, and O. Schel, "Probabilistic routing in intermittently connected networks," SIGMOBILE Mob. Comput. Commun. Rev., vol. 7, no. 3, pp. 19-20, 2003.

[8] J. Burgess, B. Gallagher, D. Jensen et al., "MaxProp: Routing for Vehicle-Based Disruption-Tolerant Networks." pp. 1-11.

[9] Q. Li, and D. Rus, "Sending messages to mobile users in disconnected ad-hoc wireless networks," in Proceedings of the 6th annual international conference on Mobile computing and networking, Boston, Massachusetts, United States, 2000.

[10]A. Kernen, J. Ott, and T. Kinen, "The ONE simulator for DTN protocol evaluation," in Proceedings of the 2nd International Conference on Simulation Tools and Techniques, Rome, Italy, 2009, pp. 1-10.

Harminder S. Bindra received a Bachelor of Engineering degree in Computer Science from P.T.U in 1999, and M.Tech. Degree in Information Technology from AAIDU, Allahabad, India in 2006. Since August 2009 he has joined as a research scholar in National Institute of Technology, Jalandhar. His research interests are in Ad hoc Networks and Delay Tolerant Networks (DTNs).

A. L. Sangal is professor in the Department of Computer Science and Engineering, at Dr B.R. Ambedkar National Institute of Technology Jalandhar (Punjab), India since 2008. He obtained his B.Tech Degree from Panjab Engineering College Chandigarh, India in the year 1985 and Master Degree in the year 1991 from Thapar Institute of Engineering \& Technology Patiala (Punjab), India and $\mathrm{PhD}$ in the field of Scientific Computing from Dr B.R. Ambedkar National Institute of Technology Jalandhar (Punjab), India in 2008. He has served as a faculty at Thapar Institute of Engineering \& Technology Patiala and Dr B.R. Ambedkar National Institute of Technology Jalandhar and served as Head of the Department of Computer Science and Engineering since 1992-2006. He has published many research papers in the field of Scientific Computing and Wireless Networks in International Journal and Conferences and presently guiding six Research Scholars of PhD / M.Tech. 